\title{
Laser Direct Ablation for Patterning Printed Wiring Boards Using Ultra- fast Lasers and High Speed Beam Delivery Architectures
}

\author{
Hisashi MATSUMOTO ${ }^{{ }_{1}}$, Mark UNRATH ${ }^{{ }_{1}}$, Haibin ZHANG $^{{ }^{* 1}}$ and Bob HAINSEY ${ }^{{ }^{* 1}}$ \\ ${ }^{* 1}$ Electro Scientific Industries, Inc. 13900 NW Science Park Dr. Portland, OR 97229, USA \\ E-mail: matsumoh@esi.com
}

\begin{abstract}
Laser Direct Ablation (LDA) for patterning printed wiring boards (PWB) is a new technology in the industry. Unlike the Laser Direct Imaging (LDI) method, this unique approach to remove the dielectric material by laser and embed the signal trace lines into the PWB allows the formation of recessed signals to below $10 \mu \mathrm{m}(4 \mu \mathrm{m}$ demonstrated) and the creation of near padless vias to facilitate a reduction in both package size and format $[4,5]$. We have developed a vector scanning based laser system for this application using a unique beam steering technology combined with a high repetition rate ultrafast laser [1 - 3]. One requirement for this application is to control the width and depth of the signal lines at the micron level. The system takes advantage of a proprietary tertiary beam positioning system that uses Acousto-Optic Deflectors (AODs) in addition to the conventional galvanometers and linear stages, and the scheme allows not only the accurate and high speed beam delivery but also precise material removal. This paper will introduce this new technology in fabricating high density interconnect circuit patterns, the laser application requirement, and system architecture for the application.
\end{abstract}

DOI: 10.2961/jlmn.2013.03.0022

Keywords: Laser Direct Ablation, Laser Microfabrication System, Beam Delivery, Ultrafast Laser Materials Processing, Printed Wiring Boards

\section{Introduction}

Recent technology advances in many different types of electronics devices are quite astonishing. As we the consumers continue to demand next generation devices to be smaller, lighter, faster and cheaper, and to have more functionalities, designers and manufacturers are pushing the technologies forward to meet consumers' expectations. The circuit designs of multi-layer PWB for those devices become more complex, require increased wiring density, and often increased layer count. Whether reducing the layer count by increasing the circuit density to cut cost, or maintaining the layer count and increasing the density to add more functionality, either way, maximizing the signal routing density is the biggest challenge. It requires reducing the width of signal lines and spacing as well as reducing the size of the capture pads.

We have developed a vector scanning based laser system for the application using a unique beam steering technology combined with a high repetition rate ultrafast laser $[1-3]$. The system takes advantage of a proprietary tertiary beam positioning system that uses Acousto-Optic Deflectors (AODs) in addition to conventional galvanometers (galvos) and linear stages. The scheme allows not only accurate and high speed beam delivery but also precise material removal.

While maintaining required signal integrity, this innovative technology utilizes laser ablation to form electrical paths for signal propagation within the dielectric, as opposed to conventional technologies that form signal paths above the dielectric $[4,5]$. With this technology, the package manufacturers can take advantage of reduced lines/pads geometries, improved adhesion of the copper traces, improved electrical performance by better control of impedance and reduced crosstalk, and further, bypasses photo-lithographical process steps. This represents a breakthrough for substrate manufacturing.

\section{Applications}

The target application is multi-layer PWB patterning, particularly for IC packaging (ICP) substrates. Typical line width/spacing for such substrate is $15 \mu \mathrm{m}$, and the state of the art design uses $12 \mu \mathrm{m}$. The target smallest line width is less than $15 \mu \mathrm{m}$. The new concept of embedding trace lines allows to control the volume of the trace line and allows the signal line to be sandwiched between ground lines. The ground lines can be wider and deeper. The signal line can be connected to capture pad with a gradual change of the line width. The system requirements for the applications are:

- Dynamical line width and depth control

- $\mathrm{X} / \mathrm{Y}$ precision: $\leq 1 \mu \mathrm{m}$ local

- Depth: $< \pm 10 \%$ of nominal

- Minimum width: $\leq 15 \mu \mathrm{m}$ ( $\leq 25 \mu \mathrm{m}$ for non ICP).

Fig. 1 illustrates the differences between the current lamination process with semi-additive plating (SAP) and the LDA process. In the figures, dielectric resin (yellow) is laminated on a copper layer (green) with capture pads (orange). The photolithographic process (step 4) to form the circuit pattern is replaced by the LDA patterning process (step c). With the currently used dielectric films, the whole panel needs to be plated after the LDA patterning (step e). The planarization / etch process (step f) will be added to grind off and etch away the excessive copper from the surface to define the circuit board pattern. 


\begin{tabular}{|c|c|c|c|}
\hline Process Step & \begin{tabular}{|l|} 
Semi Additive \\
Process
\end{tabular} & LDA Process & Process Step \\
\hline 1. Laminate Dielectric & 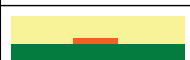 & 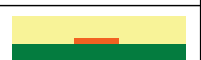 & \begin{tabular}{|l|l|} 
a. Laminate Dielectric \\
\end{tabular} \\
\hline \multirow[t]{2}{*}{ 2. Laser Drill } & & 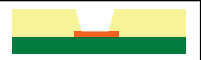 & b. Laser Drill \\
\hline & & 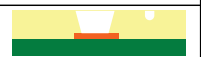 & c. LDA patterning \\
\hline $\begin{array}{l}\text { 3. Desmear I } \\
\text { Electroless Cu }\end{array}$ & & $\sqrt{2}$ & $\begin{array}{l}\text { d. Desmear / } \\
\text { Electroless Cu }\end{array}$ \\
\hline \multicolumn{4}{|l|}{$\begin{array}{l}\text { 4. Apply Resist/Photo } \\
\text { exposure/development }\end{array}$} \\
\hline 5. Pattern Plate & & - & e. Panel Plate \\
\hline \multicolumn{4}{|l|}{ 6. Strip Resist } \\
\hline 7. Etch & & $7 \cdot$ & $\begin{array}{l}\text { f. Planarization } / \\
\text { Etch }\end{array}$ \\
\hline
\end{tabular}

Fig. 1 Comparison of lamination process using semi-additive plating process and LDA process

\section{Laser System Approaches for LDA application}

There are three types of system approaches to create the circuit pattern by removing dielectric resin materials.

\subsection{Mask projection system}

The first approach uses a mask to project the whole circuit pattern on to the dielectric resin via a high power laser, typically an Excimer laser.

Pros:

- Flat and uniform dose makes precise material removal for excellent depth control, works particularly well when the signal lines cross.

- Process time independent of the circuit pattern/density

Cons:

- High cost and long lead time for the mask

- Not flexible for circuit pattern design changes

- Requires long setup time to switch to different product to process

- Not utilizing laser power efficiently

- Requires multiple masks to process different depth features in the dielectric layer

\subsection{Raster Scanning System}

The second approach scans the beam over the substrate line by line (raster) - the architecture of choice of many Laser Direct Imaging (LDI) systems [6]. The beam is scanned through a focusing lens via a fast beam steering mechanism such as a polygon mirror, and modulated on and off quickly according to the circuit pattern.

Pros:

- Maskless process

- Flexible for circuit pattern design changes

- Easy to switch to different product to process

- Process time independent of circuit pattern

Cons:

- Need to scan entire area in most case

- Ineffective if the circuit pattern is not dense across the entire PWB

\subsection{Vector Scanning System}

The last approach scans the beam where the pattern needs to be formed rather than scanning the entire area.
Pros:

- Maskless process

- Flexible for circuit pattern design change

- $\quad$ Easy to switch to different products to process

- Flexible to form different depth features

- Effective since scanning only required area

Cons:

- Process time depends on the circuit pattern

- Requires complex control mechanism

\section{System Architecture}

Fig. 2 illustrates the general architecture of the system, which is based on conventional galvanometer scanner deflecting the beam over a stationary sample. After one package process is completed, the work piece will be moved to the next processing area by $\mathrm{X} / \mathrm{Y}$ translation stage (often referred to as "step-and-repeat mode"). Our unique approach adds "tertiary" beam positioning technology, acousto-optic deflectors (AODs) to meet the high throughput and process requirements. Two AODs are placed orthogonally to generate the angle to rapidly translate the beam in $\mathrm{X} / \mathrm{Y}$ axis through the scan lens. It provides the capability to not only rapidly vary the position but also power. In a similar fashion to the traditional compound beam positioning used in galvos and linear motors scheme, where the two beam positioning systems are synchronized to form a coordinated beam trajectory $[7,8,9]$, the combination of AODs and galvos allows the process beam trajectory to be profiled at high speeds that exceed the bandwidth and acceleration capabilities of the galvos themselves. The galvos trajectories are profiled to provide the majority of the required beam deflection, with the AODs commanded to provide additional deflection and to correct for galvo tracking errors.

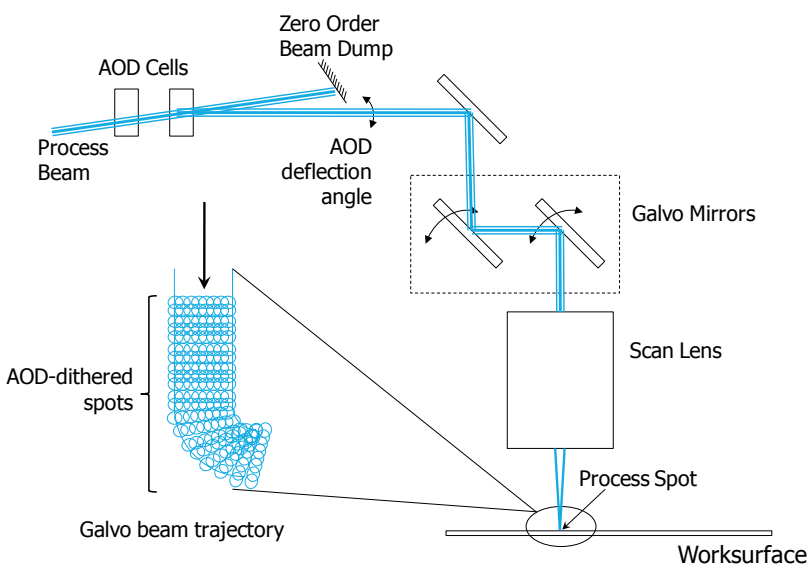

Fig. 2 General Architecture of the high speed beam delivery scheme [10]

There are many systems/architectures available that can scan the beam faster than $10 \mathrm{~m} / \mathrm{sec}$. However, such high speed scanning is achieved only for a straight line. When turning tight corners, acceleration and bandwidth become the dominating issues. Typical galvo control is limited to about 2,000 to $4,000 \mathrm{~g}$ 's at the works surface, with a bandwidth of around $2-3 \mathrm{kHz}$. With this new architecture, these limits are basically removed - effectively, with acceleration of 1,000,000 g's and bandwidth of $1 \mathrm{MHz}$. The ac- 
celeration and bandwidth constraints affect velocity indirectly. An example of the capabilities of the new architecture is shown in Fig. 3. The addition of AODs enables work surface beam velocities exceeding $2 \mathrm{~m} / \mathrm{sec}$ with no inherent limitations on turn radius, while maintaining excellent positioning repeatability.

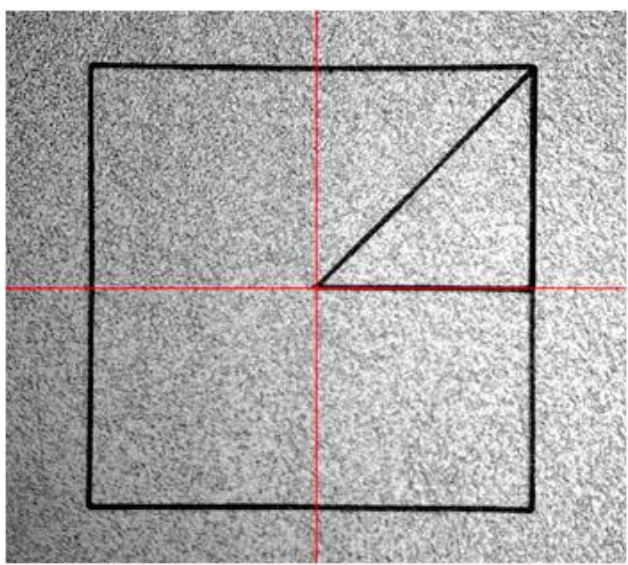

Fig. 3 Test pattern processed at $4 \mathrm{~m} / \mathrm{sec}(1 \mathrm{~mm}$ square, $25 \mu \mathrm{m}$ width)

It also provides real-time power control so that power can be maintained/modified for the same/different width/depth. When the beam is deflected orthogonal with respect to the galvo beam trajectory (dither), width of the line can be dynamically changed. Note that a key point to take advantage of this architecture is to have a high enough laser Pulse Repetition Frequency (PRF) so that the pulse overlap is sufficient.

\section{Laser Material Process}

Typical dielectric materials used for the packaging substrate are silica particle filled Epoxy resins, such as ABF from Ajinomoto. The thickness of the material is $\sim 30-35 \mu \mathrm{m}$. For widely adapted laser via drilling applications, lasers are typically either pulsed $\mathrm{CO}_{2}$ lasers or nanosecond (ns) solid state UV lasers. Due to the ever shrinking feature size requirements, shorter wavelength lasers have a significant advantage.

Ablation rate of organic polymers using UV laser is commonly characterized by incident fluence, threshold fluence and absorption coefficient based on the use of the Beer-Lambert law. For characterizing ablation process by moving Gaussian beam, we use the dose fluence, $\theta_{D S}$, defined as the cumulative fluence over the $1 / \mathrm{e}^{2}$ spot area, $\theta$ :

$$
\begin{aligned}
& \theta_{D S}=N_{\text {Spot }} \cdot \theta \cdot \text { geometric_factor } \\
& N_{\text {Spot }}=\frac{2 \omega_{0}}{\delta}
\end{aligned}
$$

$$
\text { geometric_factor }=\frac{\delta}{2 \omega_{0}} \cdot \sum_{n=-\infty}^{\infty}{ }^{-2} \frac{\delta^{2}}{\omega_{0}^{2}} \cdot n^{2}
$$

where $N_{\text {spot }}$ is the number of pulses over the spot area, $\omega_{0}$ is the $1 / \mathrm{e}^{2}$ spot radius and $\delta$ is the spot to spot distance. For a pulse overlap greater than approximately $60 \%$ (or $\left.\delta / 2 \omega_{0}<0.6\right)$, the dose fluence becomes flat with geometric factor of $\sim 0.627$ and can be generalized to:

$$
\theta_{D S}=2 \cdot 0.627 \cdot \frac{P}{\pi \cdot V \cdot \omega_{0}}
$$

where $P$ is the laser average power, and $V$ is the beam scanning velocity $(P / V$ is the linear dose).

We use fluence over the $1 / \mathrm{e}^{2}$ spot area for width characterization and center fluence of the Gaussian spot, which is twice as high as the $1 / \mathrm{e}^{2}$ spot area, for depth characterization.

$$
\theta_{D C}=2 \cdot \theta_{D S}
$$

If a constant fluence threshold exists, the spot diameter in the material, $R$, can be estimated via optical spot size, peak power, $\mathrm{P}_{0}$, and fluence [11].

$$
\begin{aligned}
& I(r)=I_{0} \exp \left(-\frac{2 r^{2}}{\omega_{0}^{2}}\right) \\
& I_{t h}=I(r=R)=I_{0} \exp \left(-\frac{2 R^{2}}{\omega_{0}^{2}}\right) \\
& R^{2}=\frac{\omega_{0}^{2}}{2} \ln \left(\frac{I_{0}}{I_{t h}}\right)
\end{aligned}
$$

Taking into the simple linear equation for fitting,

$$
R^{2}=k+b \cdot \ln \left(P_{0}\right)
$$

The slope, $k$ is

$$
k \equiv \frac{\omega_{0}^{2}}{2} \rightarrow \omega_{0}=\sqrt{2 k}
$$

And the $\mathrm{y}$ intercept, $b$ is

$$
\begin{aligned}
& b \equiv \frac{\omega_{0}^{2}}{2} \ln \left(\frac{2}{\pi \omega_{0}^{2} I_{t h} /[\text { watt }]}\right) \rightarrow \\
& I_{t h} /[\text { watt }]=\frac{2}{\pi \omega_{0}^{2}} \exp \left(-\frac{2 b}{\omega_{0}^{2}}\right)
\end{aligned}
$$

Replacing R with Width, Fig. 4 shows the example of two fits of equation (11) using two different optics configurations to vary the focused spot size.

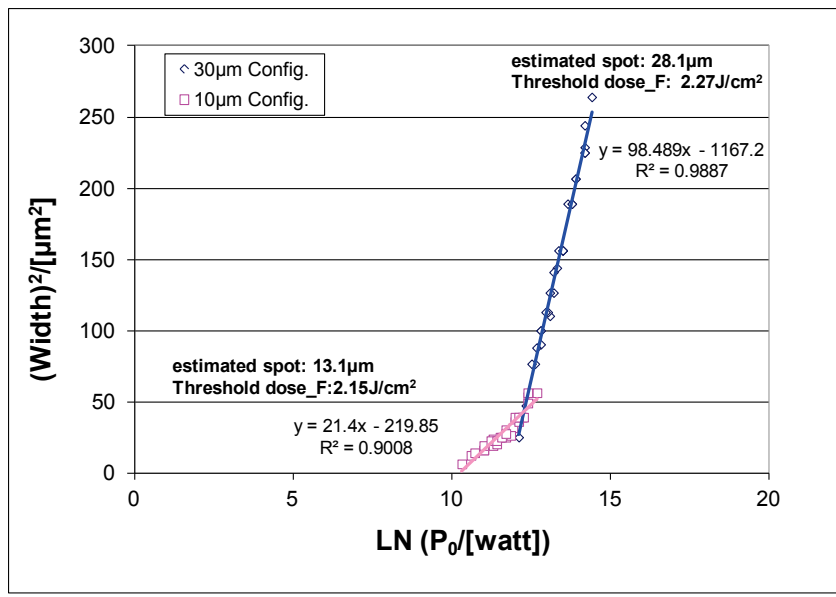

Fig. 4 Example of fits using two different optics configuration varying power and velocity using ps UV laser 
The extracted threshold fluence is approximately the same for both spot sizes as expected.

Fig. 5 is an example image of a laser scribed line formed in the GX-13 ABF resin.

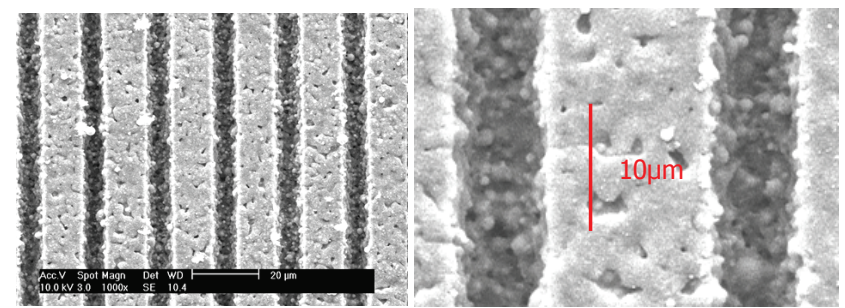

Fig. 5 Scanning Electron Microscope images of processed resin

The line width and depth was measured with a light microscope. Measuring the depth with a light microscope is very subjective and time-consuming. Fig. 6 shows alternative methods: Using a cross-sectional view is more accurate; however, it takes even more time. On the other hand, surface profilometers such as 3D laser scanning microscopes are an excellent tool to measure wide areas of width and depth. Some of these instruments can export height data of each pixel making it possible to analyze data thoroughly and automatically. Having such a metrology tool is crucial for checking the performance of the motion control system as well as understanding process capability.
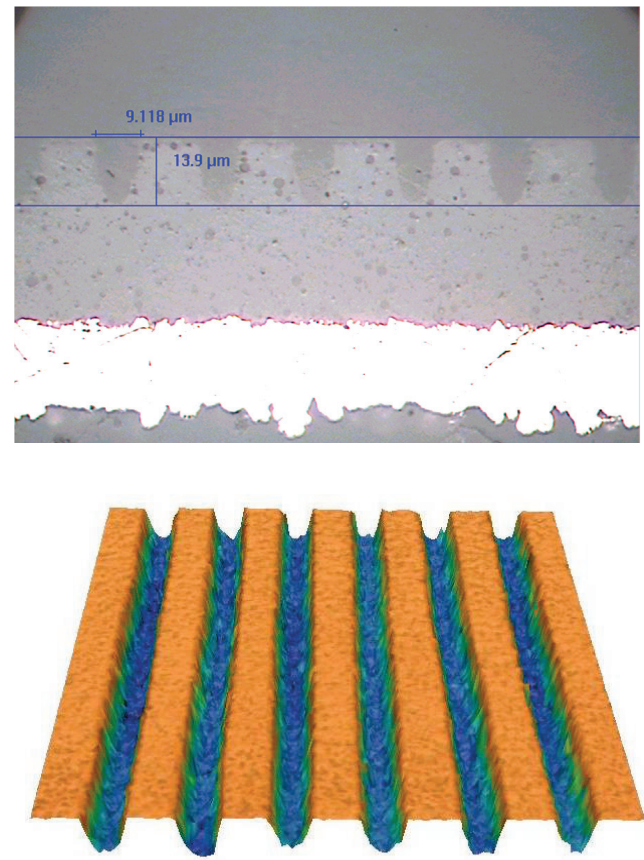

Fig. 6 Light microscope image of cross-sectioned sample (above), and 3D scanning image by Violet Laser Scanning Microscope (below). White part in the crosssection image is copper layer.

Fig. 7 shows linear dose fluence vs. ablation depth at various velocities and powers using $29 \mathrm{~ns}$ and 50 picosecond (ps) UV lasers $(355 \mathrm{~nm})$. Spot size was varied by defocusing the beam. Power and velocity range for the ns laser were $0.1-0.2 \mathrm{~W}$ at $70 \mathrm{kHz}$ and $70-160 \mathrm{~mm} / \mathrm{sec}$, and $0.25-$ $0.5 \mathrm{~W}$ at $80 \mathrm{MHz}$ and $250-1000 \mathrm{~mm} / \mathrm{sec}$ for the ps laser. The generic trend roughly matches for both lasers although operating range of pulse energy and PRF are quite different.

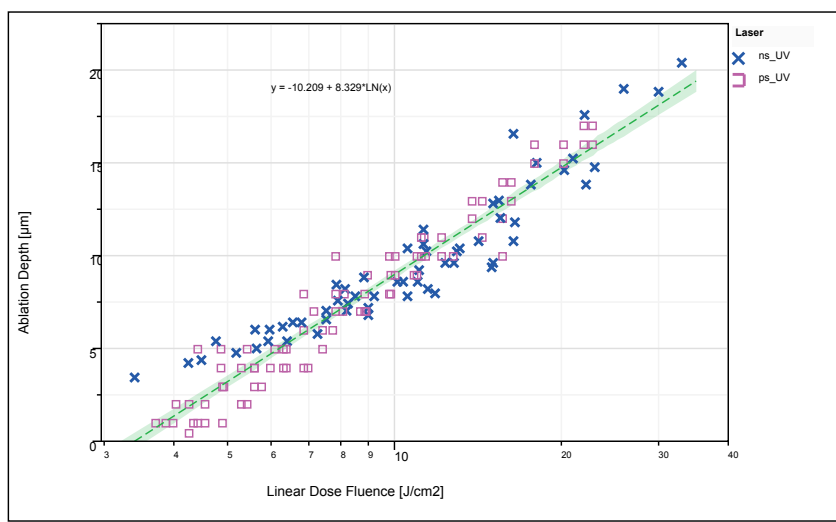

Fig. 7 Ablation depth vs. linear dose fluence with ns and ps UV lasers

Fig. 8 is the approximated ablation depth per pulse based on the number of pulses over the spot area with the beam moving. The ablation of material becomes inefficient once the beam center fluence drops below approximately $200 \mathrm{~mJ} / \mathrm{cm}^{2}$ for the ns laser and approximately $10 \mathrm{~mJ} / \mathrm{cm}^{2}$ for the ps laser.

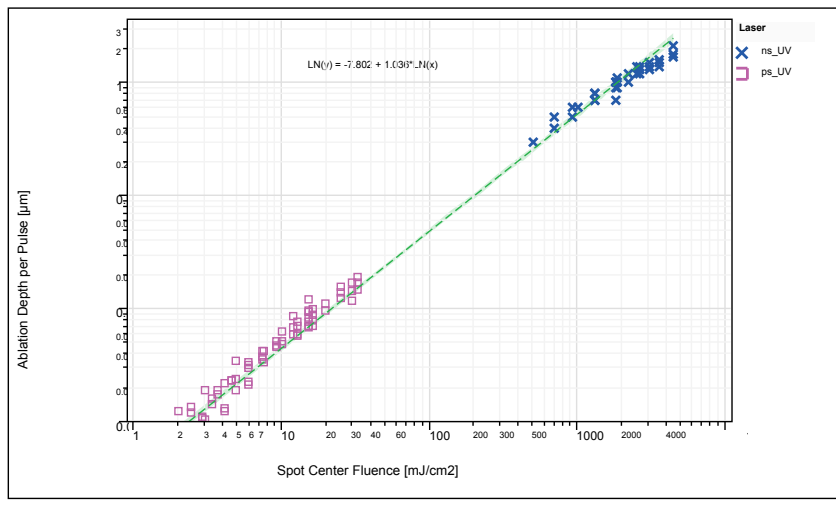

Fig. 8 Ablation depth per pulse vs. spot center fluence with ns and ps UV lasers

Fig. 9 shows the measured width and estimated spot size ratio at different dose fluences. The shaded area shows the $95 \%$ confidence range of the fitted equations. As seen in the figure, there are many outliers for the fit but some trends are nevertheless obvious. Changing the spot size by defocusing the beam is most convenient; however, it introduces complexities in estimating the effective spot diameter accurately. Our calculation is based on a perfectly collimated beam focused without astigmatism/asymmetry error and having the same $\mathrm{M}^{2}$ value for both axes. Instead of changing beam size, width can be better controlled by using a fixed spot size at a single $\mathrm{Z}$ plane (around focal plane), and by dithering the beam orthogonal to the moving direction. The new AOD technology does just that with a well controlled process for arbitrary line width. 


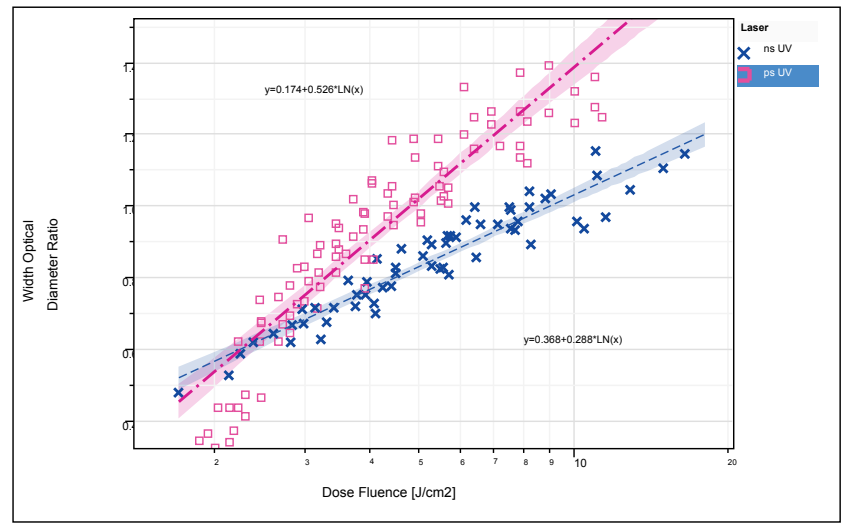

Fig. 9 Measured width and estimated spot diameter ratio vs. Dose Fluence

Most polymers tend to decompose before evaporation. Long chains of molecules are cut into fragments before they are able to leave the bulk material. Thermal activation of the ablation appears as a typical "Arrhenius tail" instead of the definite ablation threshold [12]. The threshold fluence decreases by increasing the absorption coefficient, irrespective of whether this is related to a decrease in laser wavelength, the addition of dopants, to the generation of defects, or by decreasing the pulse duration. With shorter pulses, the spatial dissipation of the excitation energy is reduced and the threshold fluence is lower. This observation can be related to a decrease in heat penetration depth and/or an increase in absorption coefficient due to multiphoton excitation [13]. The short duration (ps), high peak power and higher PRF lasers are ideal sources for the application in order to utilize the high speed beam delivery scheme. Pulse energy can be lower as long as the fluence exceeds the effective threshold. Therefore, a picosecond mode locked laser that outputs $>4 \mathrm{~W}$ at $10 \mathrm{MHz}$ to $100+\mathrm{MHz}$, can be used to take advantage of the fast beam delivery scheme and still meet the process requirement.

\section{Result}

Fig. 10 shows an example of the circuit pattern generated with our high speed beam delivery scheme and the high PRF ps laser. The trace lines were formed by dithering the beam. The pads and the large ground planes were formed by raster scanning the beam locally. The dose fluence of connecting points between pads and lines is tightly controlled avoid the double exposure effects.

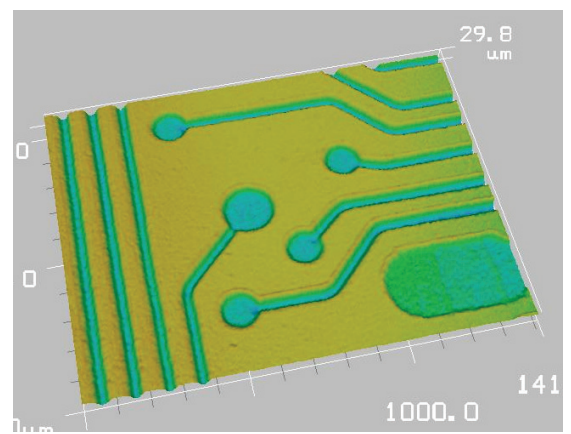

Fig. 10 3D image of patterned circuit using violet laser scanning microscope
Fig. 11 and 12 show some examples of line width and spacing capability. A $7 \mu \mathrm{m}$ line width/spacing sample was processed at $4 \mathrm{~m} / \mathrm{sec}$ velocity at a 1:1 depth/width aspect ratio. A $4 \mu \mathrm{m}$ line width/spacing has been demonstrated as the minimum feature size of the system.

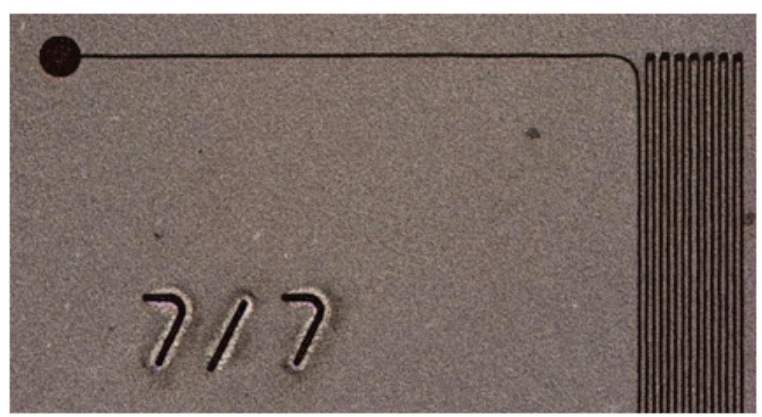

Fig. 11 Small line width/spacing demonstration

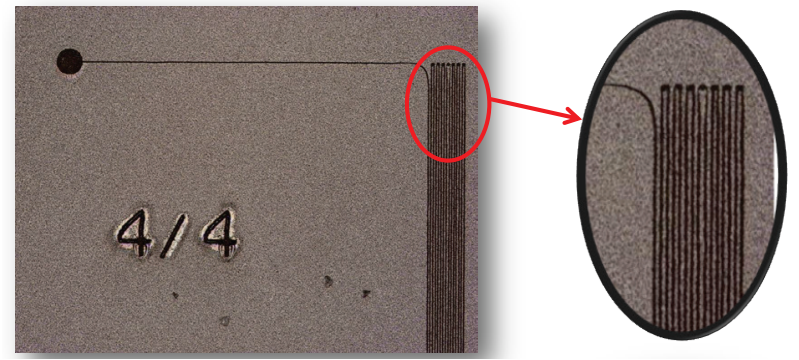

Fig. 12 Smallest line width/spacing demonstrated

Fig. 13 and 14 illustrate the variation of width and depth for lines nominally $23 \mu \mathrm{m}$ wide and $18 \mu \mathrm{m}$ deep. The limits shown in the figures are $\pm 10 \%$ from nominal for the line width, and $\pm 15 \%$ for the line depth. The material used was not ABF material (DuPont DC) and had more depth variation.

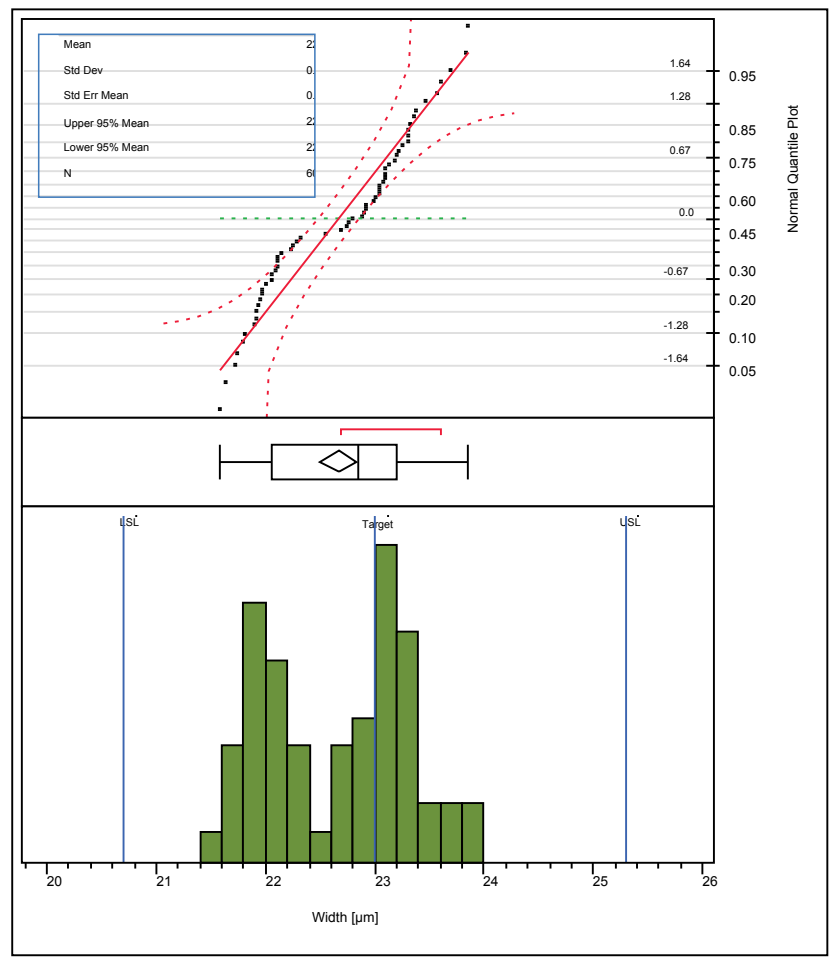

Fig. 13 Histogram of line width 


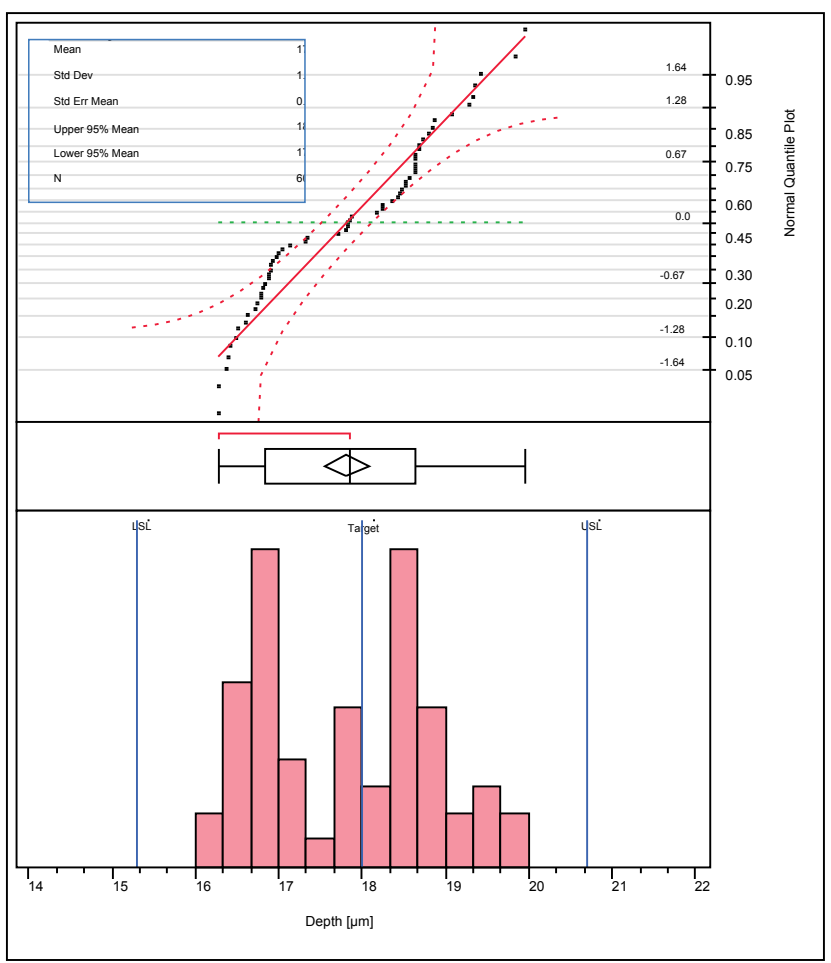

Fig. 14 Histogram of line depth

\section{Summary}

We introduced a new architecture for fabricating high density interconnects circuit patterns. To meet the requirements of cost effective scaling to higher density PWB design, a new beam delivery scheme and system using acousto-optic deflectors has been developed and introduced. The addition of AODs enables work surface beam velocities exceeding $2 \mathrm{~m} / \mathrm{sec}$ with no inherent limitations on turn radius, while maintaining excellent positioning repeatability, which is not possible using traditional vector architectures. Based on the laser/material process, mode locked ps lasers operating at high PRF have been found suitable to best take advantage of this fast beam delivery scheme.

\section{References}

[1] W. Cheng, M. Unrath, Laser Structuring Technology for DC, Proceeding of ECWC 2011, (Conference Proceedings)

[2] M. Unrath, Optomechatronic Applications: Industrial Precision Laser Processing, Invited talk at International Symposium on OptoMechatronic Technologies, (2012)

[3] H. Zhang, H. Matsumoto, G. Simenson, Q. Xu, M. Unrath, M. Orrick, M. Darwin and B. Hainsey, Laser micro-fabrication systems for consumer electronics device manufacturing, Proceeding of CLEO 2013, (Conference Proceedings)

[4] R. Huemoeller, S. Rusli, S. Chiang, T. Y. Chen, D. Baron, L Brandt and B. Roelfs, Unveiling the Next Generation in Substrate Technology, SMTA: Pan Pacific Symposium Proceedings, (2007) (Conference Proceedings)

[5] R. Huemoeller, S. Rusli, S. K. Kuchibhotla, L. Wojcik S. Appasamy and K. Jain, Unveiling the Next Generation in Integrated Circuit Substrate Circuit Formation, CircuiTree, June 2007, (2007), pp. 12-18

[6] P. Blatt, Laser Direct Imaging Benefits From Solid State Technology, Proceedings of the LANE 2007, (Conference Proceedings, available from Coherent site)

[7] D. Cutler, R. Pailthorp, M. Unrath, T. Richardson, A. Cable, Multi-tool positioning system, US 5,847,960, (Patent)

[8] D. Cutler, R. Pailthorp, M. Unrath, High speed high accuracy multi-stage tool positioning system, US 5,751,585, (Patent)

[9] D. Cutler, R. Pailthorp, Apparatus and method for coordinating the movements of stages in a multi-stage multi-rate positioned system, US 5,798,927, (Patent)

[10] M. Unrath, W. Jordens, J. Ismail, H. Matsumoto, B. Linedurg, Acousto-optic deflector applications in laser processing of dielectric or other materials, US 8,404,998, (Patent)

[11]L. Zou, W. Lei, Measurement of Focused Beam Waist of UV Pulse Laser And Material Processing Threshold, ICALEO Proceedings, (2002) (Conference Proceedings)

[12] G. Raciukaitis, M. Gedvilas, Processing of Polymers by UV Picosecond Lasers, ICALEO Proceedings, (2005) (Conference Proceedings)

[13]Dieter Bäuerle, Laser Processing and Chemistry $\left(3^{\text {rd }}\right.$ edition), (Springer-Verlag Berlin Heidelberg, 2000) p236-239 (Books)

(Received: July 18, 2013, Accepted: November 27, 2013) 